\title{
Calculation of full permeability tensor for fractured anisotropic media
}

\author{
G. Lei $\cdot$ P. C. Dong $\cdot$ S. Y. Mo $\cdot$ S. Yang $\cdot$ \\ Z. S. Wu $\cdot$ S. H. Gai
}

Received: 22 April 2014/Accepted: 15 September 2014/Published online: 25 September 2014

(C) The Author(s) 2014. This article is published with open access at Springerlink.com

\begin{abstract}
Fractured reservoirs' media consists of matrix and effective fractures. The fracture distribution is complex and variable in these reservoirs with strong characters of anisotropy of reservoirs. This paper introduces a simple, fast and accurate method to test 2D tensor permeability of fractured anisotropy media. Combining numerical simulation results and experiment results, 2D tensor permeability is derived and the variation mechanism of $2 \mathrm{D}$ tensor permeability in fractured anisotropic media has been revealed. According to the polar form of elliptic equation, permeability elliptic is derived. With ellipsoidal permeability, the change law of permeability value in principal direction is studied. 3D permeability tensor model for fractured media is proposed on basis of the co-ordinate transformation principle. Based on the quantitative characterization of $3 \mathrm{D}$ tensor permeability and Gangi's permeability stress sensitivity model, a stress-dependent 3D permeability tensor mathematical model for fractured media is established. The method provides a theoretical basis for the determination of percolation parameters in fractured reservoirs, and the results are significant in understanding the fluid flow in fractured anisotropic media.
\end{abstract}

Keywords Permeability tensor experiment · Fractured anisotropic media $\cdot$ Permeability elliptic $\cdot$ Permeability stress sensitivity

G. Lei $(\bowtie) \cdot$ P. C. Dong · S. Y. Mo · S. Yang ·

Z. S. Wu - S. H. Gai

Department of Petroleum Engineering, China University

of Petroleum, Beijing, China

e-mail: leigang5000@126.com

\section{Introduction}

It is well known that fractured porous media is often anisotropic, which means permeability values depend on the direction at which it is measured. The permeability parallel to the fracture is usually greater than the perpendicular direction. Many investigators have attempted to either experimentally develop a procedure to measure directional permeabilities or to develop a mathematical model to calculate anisotropy permeability of a system.

Johnson et al. (1948; Johnson and Breston 1951) analyzed a series of oil well cores by cutting them into small horizontal plugs and observed that the permeability varies with the direction in which the plug is cut. Compared with the theory of Darcy for isotropy porous media, an extension for anisotropic media has been developed by Ferrandon (1948) upon theoretical grounds, in which permeability is represented as a symmetric tensor. Based on the study of Johnson et al. (1948; Johnson and Breston 1951) and Ferrandon's theory (1948), Scheidegger (1954) obtained the substantiation of the tensor theory. Liakopoulos (1960, 1965a, b) described the permeability in homogenous anisotropic soils by a second rank, symmetric, positive definite tensor. Chapuis (1989) studied how densification influences the anisotropy of sands and sedimentary rocks by laboratory test method, and found the anisotropy of sandstone increases with densification. Leung (1986) presented the physical and mathematical interpretation for the cross terms in the Cartesian 2D permeability tensor. Marcus (1962) and Parsons (1964) studied the laboratory results of directional permeability, and concluded that the directional permeability value of a sample is an apparent value depending on the $\mathrm{L} / \mathrm{W}$ ratio (length/width) of the sample. Masland et al. (1955), Greenkorn et al. (1964) and Asadi et al. (2000) studied anisotropic permeability by laboratory test method, and measurement model of the principal 
permeabilities of anisotropic media is developed, but the calculation of anisotropic permeability tensor is not given. Based on the laboratory test method of Asadi (Asadi 2000), Ma et al. (2013) established a new laboratory full tensor permeability test method, the new test method supposes that the pressure gradient is added to $x$ direction for forming single direction displacement, which is in contradiction with the Fig. 5b in Chen's literature (Chen et al. 1998), the pressure gradient in $y$ direction cannot be ignored for fractured anisotropic media.

Based on statistical characteristics of critically oriented fractures, Snow (1969) proposed a calculation method for permeability tensor of fractured rock masses. Oda (1985) puts forward a method using statistical theory to deduce permeability tensor. Liu et al. (2011) established 3D full permeability tensor for actual reservoir with fracture azimuth and fracture dip. Dayani et al. (2012), Bagheri et al. (2007) and Hassanpour et al. (2008) studied permeability tensor through numerical simulation method. Since fractured media has strong stress sensitivity in permeability, research on permeability tensor formulation and stress sensitivity has been reported in the literature. Metwally et al. (2010) studied permeability tensor of shale by laboratory test method, and found the relationship between anisotropy ratio and the effective pressure. Chen et al. (1998) studied stress changes of an anisotropic reservoir, and found the evolution of reservoir stress anisotropy would be influenced by the trend of permeability anisotropy. Rong et al. (2013) proposed a model of the fracture permeability tensor, and he also proposed an elastic constitutive model of rock fractures, considering fracture closure and dilation during shearing. Wong et al. (2001, 2003) proposed a flow mathematical model for stress-sensitive fractured reservoir, and he concluded that the change of fractured anisotropic permeability was caused by stress. Mina et al. (2004) and Renard et al. (2001) studied the change characteristics of the stress-sensitive fracture permeability using numerical experiment method with rock stretching and shear deformation.

In above literatures, the permeability tensor measurement method and theory calculation method just suit the permeability in principal direction, which means that how to study permeability accurately in anisotropic media is still a problem. In this paper, a new laboratory $2 \mathrm{D}$ tensor permeability test method with the pressure gradient in $x$ and $y$ directions is established, and 2D tensor permeability for a fractured media is derived with the test results and numerical simulation results. Through co-ordinate transformation, we get permeability elliptic equation of fractured anisotropic media. Based on the quantitative characterization of 3D tensor permeability and Gangi's (1978) permeability stress sensitivity model, the mathematical model of the 3D tensor permeability for fractured anisotropic media is established.

\section{Experimental work}

The 2D tensor permeability theory

For single-phase flow in fractures system, the velocity equations can be written as

$\left\{\begin{array}{l}v_{x}=\frac{Q_{x}}{A}=\frac{k_{x x}}{\mu} \frac{\partial p}{\partial x}+\frac{k_{x y}}{\mu} \frac{\partial p}{\partial y}+\frac{k_{x z}}{\mu} \frac{\partial p}{\partial z} \\ v_{y}=\frac{Q_{y}}{A}=\frac{k_{y x}}{\mu} \frac{\partial p}{\partial x}+\frac{k_{y y}}{\mu} \frac{\partial p}{\partial y}+\frac{k_{y z}}{\mu} \frac{\partial p}{\partial z} \\ v_{z}=\frac{Q_{z}}{A}=\frac{k_{z x}}{\mu} \frac{\partial p}{\partial x}+\frac{k_{z y}}{\mu} \frac{\partial p}{\partial y}+\frac{k_{z z}}{\mu} \frac{\partial p}{\partial z}\end{array}\right.$

where $v_{x}, v_{y}$ and $v_{z}$ are flow velocities in $x, y$ and $z$ directions of fluid, $\mathrm{cm} / \mathrm{s} ; Q_{x}, Q_{y}$ and $Q_{z}$ are flow rate in $x, y$ and $z$ directions, $\mathrm{cm}^{3} / \mathrm{s} ; \mu$ is the viscosity of fluid, $\mathrm{mPa} \mathrm{s} ; A$ is the cross-sectional area, $\mathrm{cm}^{2} ; k_{x x}, k_{x y}, k_{x z}, k_{y x}, k_{y y}, k_{y z}, k_{z x}$, $k_{z y}, k_{z z}$ are $3 \mathrm{D}$ full permeability tensors, $\mu \mathrm{m}^{2} ; p$ is pressure, MPa.

If pressure gradient is added to $x$ and $y$ directions for forming 2D displacement and the boundary in $y$ direction is closed, Eq. (1) can be simplified to Eq. (2):

$$
\left\{\begin{array}{l}
v_{x}=\frac{Q_{x}}{A}=\frac{k_{x x}}{\mu} \frac{\partial p}{\partial x}+\frac{k_{x y}}{\mu} \frac{\partial p}{\partial y} \\
v_{y}=\frac{Q_{y}}{A}=\frac{k_{y x}}{\mu} \frac{\partial p}{\partial x}+\frac{k_{y y}}{\mu} \frac{\partial p}{\partial y} \\
v_{z}=\frac{Q_{z}}{A}=\frac{k_{z x}}{\mu} \frac{\partial p}{\partial x}+\frac{k_{z y}}{\mu} \frac{\partial p}{\partial y} .
\end{array}\right.
$$

Since facture aperture is much lower than fracture length, velocity in $z$ direction can be ignored. Thus, Eq. (2) can be simplified to Eq. (3):

$$
\left\{\begin{array}{c}
v_{x}=\frac{k_{x x}}{\mu} \frac{\partial p}{\partial x}+\frac{k_{x y}}{\mu} \frac{\partial p}{\partial y} \\
v_{y}=\frac{k_{y x}}{\mu} \frac{\partial p}{\partial x}+\frac{k_{y y}}{\mu} \frac{\partial p}{\partial y} .
\end{array}\right.
$$

The Stable flow of incompressible fluids through the fracture medium can be governed by the diffusivity equation and the equation in Cartesian co-ordinates can be written as

$\frac{\partial v_{x}}{\partial x}+\frac{\partial v_{y}}{\partial y}=0$.

Substituting Eq. (3) into Eq. (4), we can get

$\frac{k_{x x}}{\mu} \frac{\partial^{2} p}{\partial x^{2}}+\frac{2 k_{x y}}{\mu} \frac{\partial^{2} p}{\partial x \partial y}+\frac{k_{y y}}{\mu} \frac{\partial^{2} p}{\partial y^{2}}=0$. 
Fig. 1 Schematic of discrete grid. a Displacement in $x$ direction, $\mathbf{b}$ displacement in $y$ direction

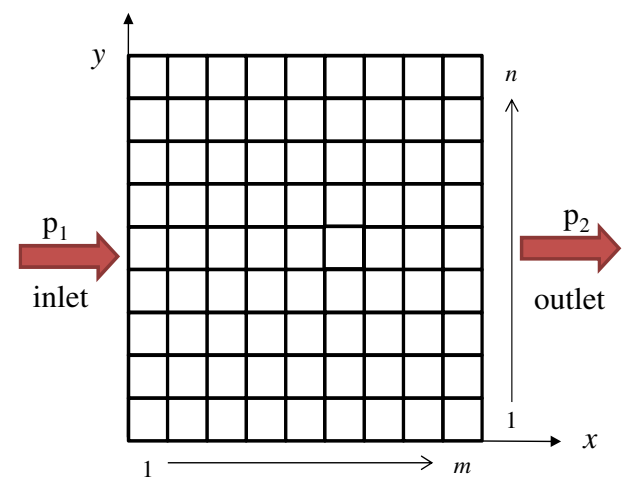

(a) Displacement in $x$ direction
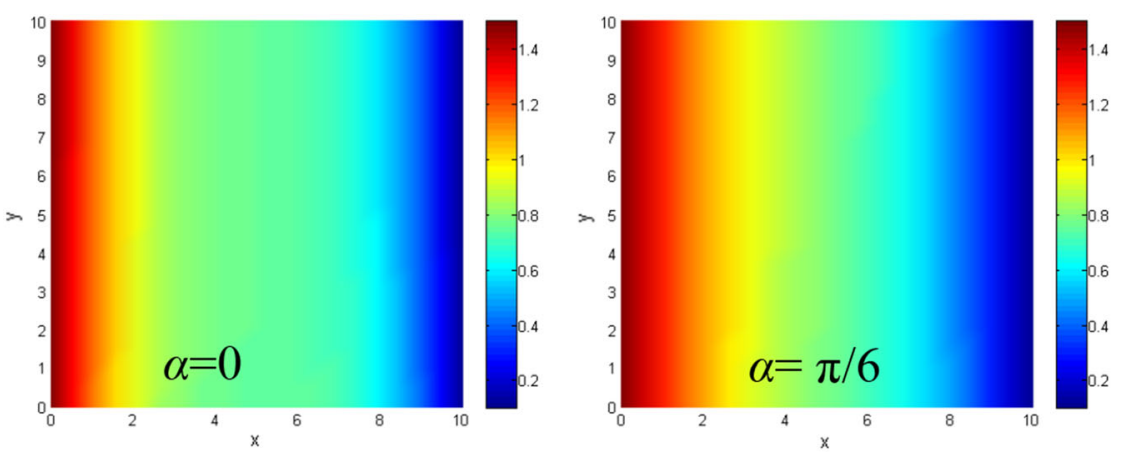

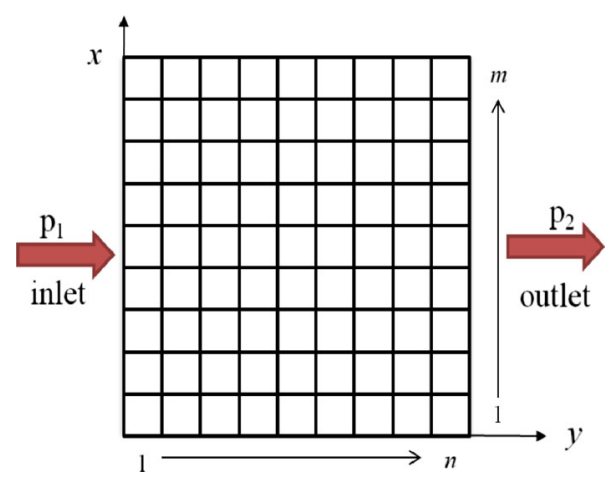

(b) Displacement in $y$ direction

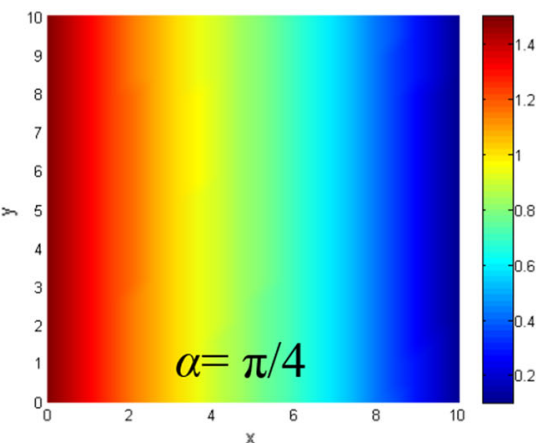

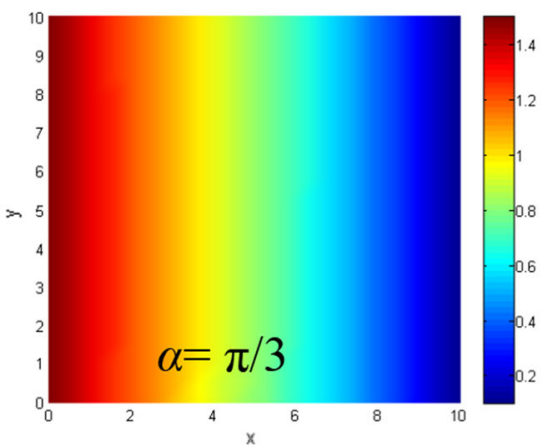

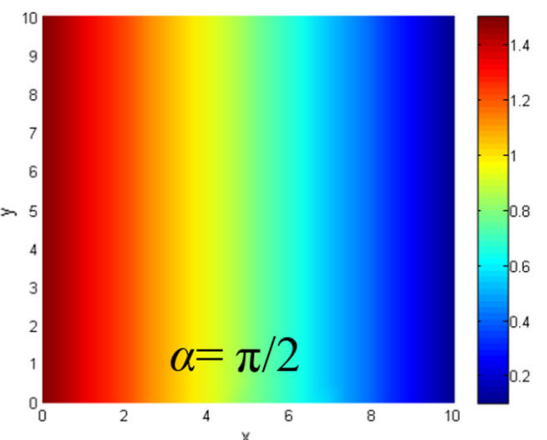

Fig. 2 Distribution drawings of pressure under different permeability fields

The boundary conditions for Eq. (5) are:

$$
\begin{aligned}
p(x=0, y) & =p_{1} ; p(x=l, y)=p_{2} ; \frac{\partial p(x, y=0)}{\partial y} \\
& =0 ; \frac{\partial p(x, y=l)}{\partial y}=0 .
\end{aligned}
$$

The whole fracture media is divided into $m \times n$ segments (The discrete grid is shown in Fig. 1). The left boundary is inlet and the right boundary is outlet. The upper and the lower boundaries are closed and impermeable. Equations (5) and (6) are solved here using finite difference method, the distributions of pressure and velocity under different permeability fields are shown in Figs. 2 and 3.

According to the generalized Darcy's law, the flow rate equation of outlet can be concluded

$$
\left\{\begin{array}{l}
Q_{x(i, m)}^{\mathrm{cal}}=A\left(\frac{k_{x x}}{\mu} \frac{p_{i, m-1}-p_{i, m}}{\Delta x}+\frac{k_{x y}}{\mu} \frac{p_{i-1, m}-p_{i, m}}{\Delta y}\right) \\
Q_{y(i, m)}^{\mathrm{cal}}=A\left(\frac{k_{y x}}{\mu} \frac{p_{i, m-1}-p_{i, m}}{\Delta x}+\frac{k_{y y}}{\mu} \frac{p_{i-1, m}-p_{i, m}}{\Delta y}\right)
\end{array}\right.
$$

and the average flow rate equations of outlet can be derived below

$\left\{\begin{array}{l}Q_{x}^{\mathrm{cal}}=\frac{1}{n} \sum_{i=1}^{n} Q_{x(i, m)}^{\mathrm{cal}}=A\left(\frac{k_{x x}}{\mu} \frac{1}{n} \sum_{i=1}^{n} \frac{p_{i, m-1}-p_{i, m}}{\Delta x}+\frac{k_{x y}}{\mu} \frac{1}{n} \frac{p_{1, m}-p_{n, m}}{\Delta y}\right) \\ Q_{y}^{\mathrm{cal}}=\frac{1}{n} \sum_{i=1}^{n} Q_{y(i, m)}^{\mathrm{cal}}=A\left(\frac{k_{y x}}{\mu} \frac{1}{n} \sum_{i=1}^{n} \frac{p_{i, m-1}-p_{i, m}}{\Delta x}+\frac{k_{y y}}{\mu} \frac{1}{n} \frac{p_{1, m}-p_{n, m}}{\Delta y}\right)\end{array}\right.$

Model length and width are $l$, percolation media is homogeneous, and single-phase fluid is water. Along with 

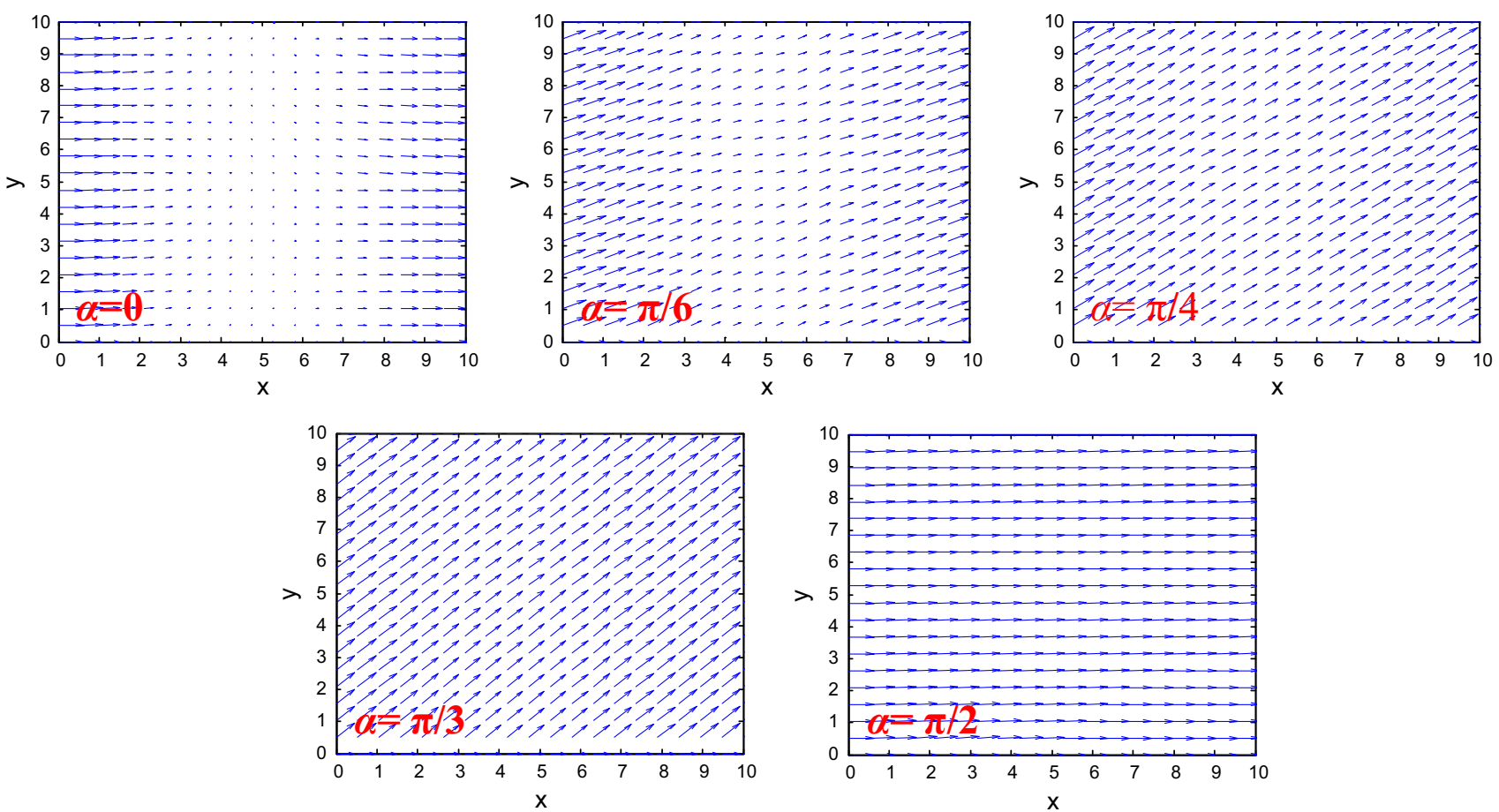

Fig. 3 Distribution drawings of velocity under different permeability fields

the outlet boundary, the flow rate of each segment in outlet boundary is non-linear. By measuring the flow rate of each segment, the rate distributions in outlet are

$$
\left\{\begin{array}{r}
Q_{x}^{\mathrm{ex}}=\frac{1}{n} \sum_{i=1}^{n} q_{i, m} \\
Q_{y}^{\mathrm{ex}}=\frac{1}{n} \sum_{i=1}^{n}\left(q_{i, m}-q_{i-1, m}\right) .
\end{array}\right.
$$

Assume $Q_{x}^{\mathrm{cal}}=Q_{x}^{\mathrm{ex}}$ and $Q_{y}^{\mathrm{cal}}=Q_{y}^{\mathrm{ex}}$, we can get the following equations

$$
\left\{\begin{array}{l}
\sum_{i=1}^{n} q_{i, m}=A\left(\frac{k_{x x}}{\mu} \sum_{i=1}^{n} \frac{p_{i, m-1}-p_{i, m}}{\Delta x}+\frac{k_{x y}}{\mu} \frac{p_{1, m}-p_{n, m}}{\Delta y}\right) \\
\sum_{i=1}^{n}\left(q_{i, m}-q_{i-1, m}\right)=A\left(\frac{k_{y x}}{\mu} \sum_{i=1}^{n} \frac{p_{i, m-1}-p_{i, m}}{\Delta x}+\frac{k_{y y}}{\mu} \frac{p_{1, m}-p_{n, m}}{\Delta y}\right) .
\end{array}\right.
$$

Then the model is rotated $\pi / 2$ and tested again, we can get the flow rate equation of outlet

$Q_{y(i, n)}^{\mathrm{cal}}=A\left(\frac{k_{y x}}{\mu} \frac{p_{i-1, n}-p_{i, n}}{\Delta x}+\frac{k_{y y}}{\mu} \frac{p_{i, n-1}-p_{i, n}}{\Delta y}\right)$.

Based on the flow rate of each segment, the rate distribution of outlet can be obtained

$Q_{y}^{\mathrm{ex}}=\frac{1}{m} \sum_{i=1}^{m} q_{i, n}$
In the same way, we can get the following equation

$\sum_{i=1}^{m} q_{i, n}=A\left(\frac{k_{y x}}{\mu} \frac{p_{1, n}-p_{m, n}}{\Delta x}+\frac{k_{y y}}{\mu} \sum_{i=1}^{m} \frac{p_{i, n-1}-p_{i, n}}{\Delta y}\right)$

combining Eqs. (10) and (13), the 2D tensor permeability can be derived.

The experimental method

The anisotropic permeability can be expressed

$[k]=\left[\begin{array}{ll}k_{x x} & k_{x y} \\ k_{y x} & k_{y y}\end{array}\right]$.

Figure $4 \mathrm{a}$ is a schematic diagram of experimental setup mainly consisting of an injection pump (ISCO), pressure sensor, a cube holder and graduated cylinders. The materials for model are sandstone outcrops. Experimental fluid is distilled water. Figure $4 \mathrm{~b}$ is a schematic diagram of the model designed for testing the anisotropy of fractures with different directions. The length of the model is $10 \mathrm{~cm}$, the width is $10 \mathrm{~cm}$ and the height is $1 \mathrm{~cm}$. The fractures in each model are parallel with $8 \mathrm{~mm}$ fracture spacing, and the angles between $x$ coordinate and fractures are $0, \pi / 6, \pi / 4, \pi / 3$ and $\pi / 2$, respectively. The properties of experimental materials are shown in Table 1. The pressure of outlet and inlet is atmospheric pressure 0.1 and $1.5 \mathrm{MPa}$, respectively. 
Fig. 4 a Schematic diagram of experimental setup, b schematic of different angles fracture distributions (a)

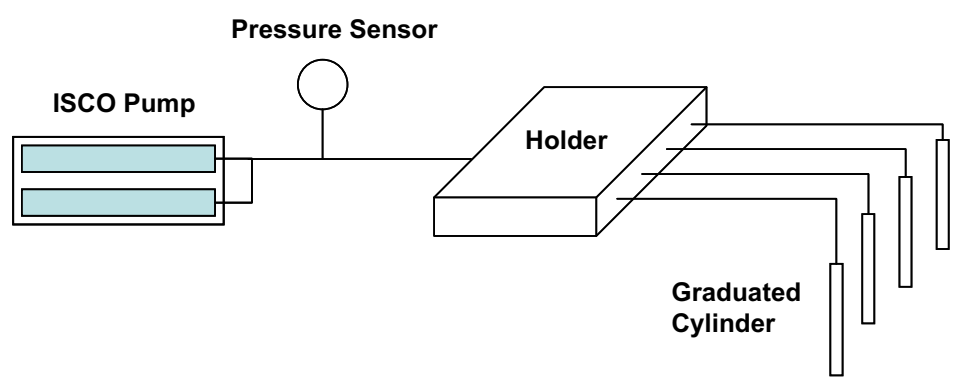

(b)

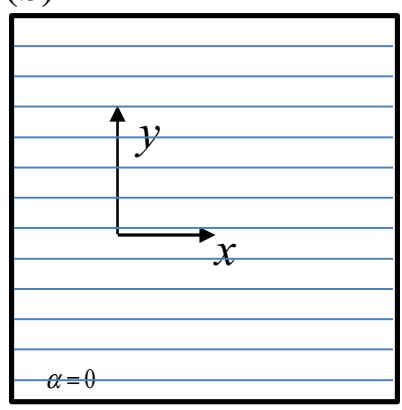

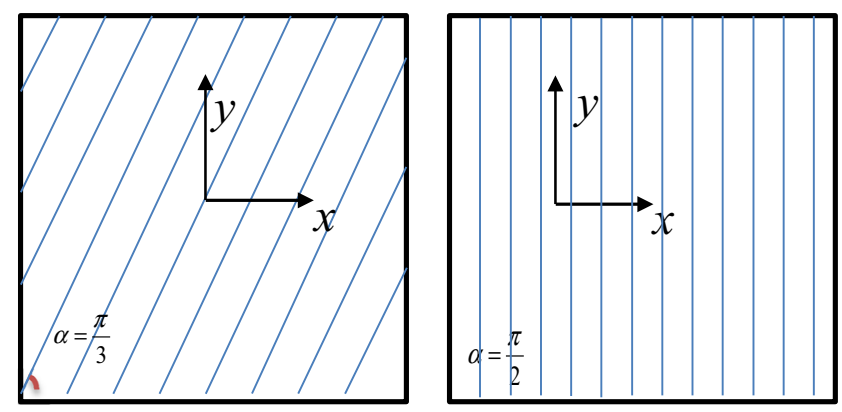

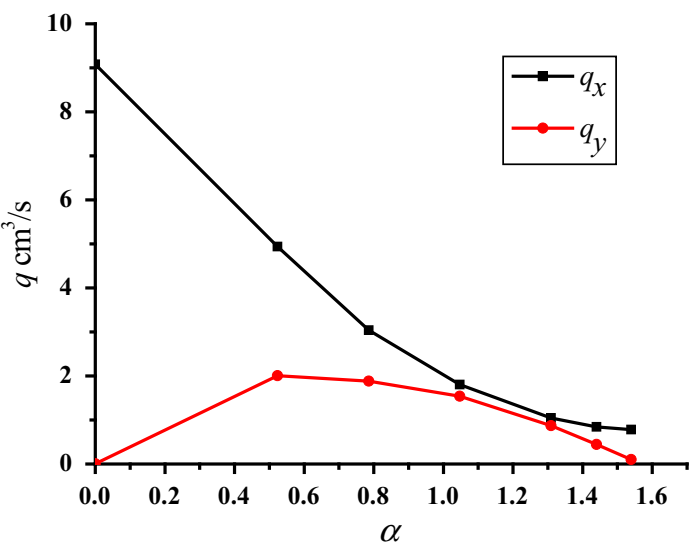

Fig. 5 Outlet flow drawing of different fracture angles

co-ordinate transformation principle, the relationship between $\left[k^{\prime}\right]$ in $2 \mathrm{D}$ and $[k]$ in $2 \mathrm{D}$ can be described

$$
\begin{aligned}
{\left[k^{\prime}\right] } & =[T][k]\left[T^{\prime}\right] \\
& =\left[\begin{array}{cc}
\cos \beta & \sin \beta \\
-\sin \beta & \cos \beta
\end{array}\right]\left[\begin{array}{ll}
k_{x x} & k_{x y} \\
k_{x y} & k_{y y}
\end{array}\right]\left[\begin{array}{cc}
\cos \beta & -\sin \beta \\
\sin \beta & \cos \beta
\end{array}\right]
\end{aligned}
$$

anisotropic model. With the increase of fracture angle $\alpha$ $(\alpha<\pi / 2)$, the flow rate in $x$ direction $q_{x}$ will decrease, however, the flow rate in $y$ direction $q_{y}$ will firstly increase then decrease.

Figure 6 shows the 2D $x^{\prime}-y^{\prime}$ and $x-y$ Cartesian co-ordinates, and the angle between the two co-ordinate systems is $\beta$. We assume the $x^{\prime}-y^{\prime}$ co-ordinate system is parallel to the direction of the permeability principal values. According to

Table 1 Properties of experimental materials

\begin{tabular}{llll}
\hline Experiment & Fracture angle & Matrix material & Fluid \\
\hline 1 & 0 & Sandstone & Distilled water \\
2 & $\pi / 6$ & Sandstone & Distilled water \\
3 & $\pi / 4$ & Sandstone & Distilled water \\
4 & $\pi / 3$ & Sandstone & Distilled water \\
5 & $\pi / 2$ & Sandstone & Distilled water \\
\hline
\end{tabular}

chained, respectively

$\left[k\left(\frac{\pi}{4}\right)\right]=\left[\begin{array}{ll}1.22 & 0.84 \\ 0.84 & 1.22\end{array}\right] ;$

$\left[k\left(\frac{\pi}{3}\right)\right]=\left[\begin{array}{cc}0.78 & 0.74 \\ 0.74 & 1.65\end{array}\right] ;\left[k\left(\frac{\pi}{2}\right)\right]=\left[\begin{array}{cc}0.35 & 0 \\ 0 & 2.08\end{array}\right]$.

or

$\left[k^{\prime}\right]=\left[\begin{array}{cc}\frac{k_{x x}+k_{y y}}{2}+\frac{k_{x x}-k_{y y}}{2} \cos 2 \beta+k_{x y} \sin 2 \beta & \frac{k_{y y}-k_{x x}}{2} \sin 2 \beta+k_{x y} \cos 2 \beta \\ \frac{k_{y y}-k_{x x}}{2} \sin 2 \beta+k_{x y} \cos 2 \beta & \frac{k_{x x}+k_{y y}}{2}-\frac{k_{x x}-k_{y y}}{2} \cos 2 \beta-k_{x y} \sin 2 \beta\end{array}\right]$.

The permeability tensor of each component can be expressed as 


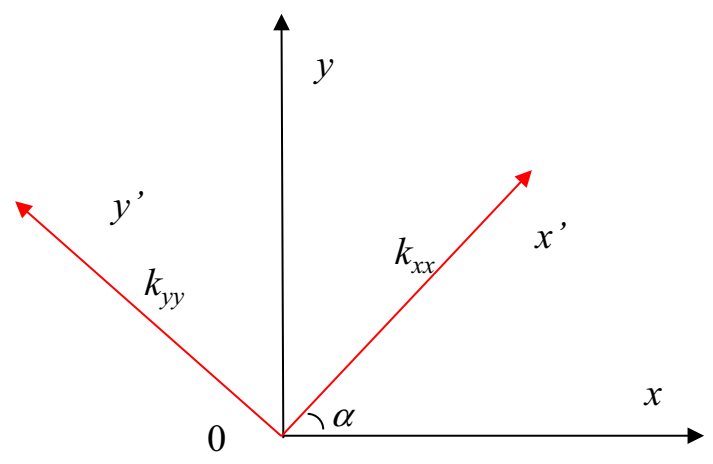

Fig. 6 Schematic of anisotropic permeability tensor in different coordinates

$\left\{\begin{array}{l}k_{x x}^{\prime}=\frac{k_{x x}+k_{y y}}{2}+\frac{k_{x x}-k_{y y}}{2} \cos 2 \beta+k_{x y} \sin 2 \beta \\ 0=\frac{k_{y y}-k_{x x}}{2} \sin 2 \beta+k_{x y} \cos 2 \beta \\ k_{y y}^{\prime}=\frac{k_{x x}+k_{y y}}{2}-\frac{k_{x x}-k_{y y}}{2} \cos 2 \beta-k_{x y} \sin 2 \beta .\end{array}\right.$

And the angle $\beta$ can be written as

$\beta=\frac{1}{2} \arctan \left(\frac{2 k_{x y}}{k_{x x}-k_{y y}}\right)$

with Eq. (16), the testing permeability tensor can be turning into the principal value co-ordinate system$$
\left\{\begin{array}{l}
{\left[k^{\prime}(\alpha=0)\right]=\left[\begin{array}{cc}
2.09 & 0 \\
0 & 0.35
\end{array}\right] ; \beta(\alpha=0)=0} \\
{\left[k^{\prime}\left(\alpha=\frac{\pi}{6}\right)\right]=\left[\begin{array}{cc}
2.09 & 0 \\
0 & 0.35
\end{array}\right] ; \beta\left(\alpha=\frac{\pi}{6}\right)=0.5201} \\
{\left[k^{\prime}\left(\alpha=\frac{\pi}{4}\right)\right]=\left[\begin{array}{cc}
2.06 & 0 \\
0 & 0.38
\end{array}\right] ; \beta\left(\alpha=\frac{\pi}{4}\right)=0.7854} \\
{\left[k^{\prime}\left(\alpha=\frac{\pi}{3}\right)\right]=\left[\begin{array}{cc}
0.36 & 0 \\
0 & 2.07
\end{array}\right] ; \beta\left(\alpha=\frac{\pi}{3}\right)=-0.5197} \\
{\left[k^{\prime}\left(\alpha=\frac{\pi}{2}\right)\right]=\left[\begin{array}{cc}
0.35 & 0 \\
0 & 2.08
\end{array}\right] ; \beta\left(\alpha=\frac{\pi}{2}\right)=0 .}
\end{array}\right.
$$

The experimental fracture angles are $0, \pi / 6, \pi / 4, \pi / 3$ and $\pi / 2$, compared with Eq. (20), the relative error Calculation formulas can be written as

$\xi_{\beta}=\left\{\begin{array}{r}\frac{1}{\alpha}|| \beta|-\alpha|=\frac{1}{\alpha}|\beta-\alpha| \quad \alpha \leq \frac{\pi}{4} \\ \frac{1}{\alpha}|| \beta\left|-\left(\frac{\pi}{2}-\alpha\right)\right|=\frac{1}{\alpha}\left|\beta+\frac{\pi}{2}-\alpha\right| \quad \frac{\pi}{4}<\alpha \leq \frac{\pi}{2} .\end{array}\right.$

The relative errors of fracture angles between measured values and theoretical values are $0,6.7 \%, 0,0.37 \%$ and 0 . Combining the formulas (17) and (20), the experimental relative errors of permeability are $\xi_{[k]}=\left|\frac{\left|\left[k^{\prime}\right]\right|-|[k]|}{|[k]|}\right|$

The relative errors are $0,0,7.01,1.87$ and $0.48 \%$.

\section{The characteristics of permeability elliptic}

Permeability elliptic of $2 \mathrm{D}$ tensor permeability

If the $x-y$ co-ordinate system is parallel to the direction of the permeability principal values, the expression of $\left[k^{\prime}\right]$ can be calculated as

$$
\begin{aligned}
{\left[k^{\prime}\right] } & =[T][k]\left[T^{\prime}\right] \\
& =\left[\begin{array}{ll}
\cos ^{2} \beta k_{x x}+\sin ^{2} \beta k_{y y} & \frac{k_{y y}-k_{x x}}{2} \sin 2 \beta \\
\frac{k_{y y}-k_{x x}}{2} \sin 2 \beta & \sin ^{2} \beta k_{x x}+\cos ^{2} \beta k_{y y}
\end{array}\right]
\end{aligned}
$$

So $k_{x x}^{\prime}=\cos ^{2} \beta k_{x x}+\sin ^{2} \beta k_{y y} ; k_{y y}^{\prime}=\cos ^{2} \beta k_{y y}+\sin ^{2} \beta k_{x x}$ according to the polar form of elliptic equation, we can get $\frac{\left(l_{x} \cos \beta\right)^{2}}{a^{2}}+\frac{\left(l_{x} \sin \beta\right)^{2}}{b^{2}}=1$

$\frac{\left(l_{y} \cos \beta\right)^{2}}{b^{2}}+\frac{\left(l_{y} \sin \beta\right)^{2}}{a^{2}}=1$

where $a=\sqrt{\frac{1}{k_{x x}}} ; b=\sqrt{\frac{1}{k_{y y}}} ; l_{x}=\sqrt{\frac{1}{k_{x x}^{\prime}}} ; l_{y}=\sqrt{\frac{1}{k_{y y}^{\prime}}}$.

As can be seen, $k_{x x}^{\prime}$ and $k_{y y}^{\prime}$ can be described with the seepage elliptic. Taking the experiment data of the first part for example, the seepage elliptic is shown in Fig. 7. The two elliptical axes are $a$ and $b$, the co-ordinates of the points are $\left(l_{x} \cos \beta, l_{x} \sin \beta\right)$ and $\left(l_{y} \cos \beta, l_{y} \sin \beta\right) . k_{x x}^{\prime}, k_{x y}^{\prime}$ and $k_{y y}^{\prime}$ vary periodically with the angle (shown in Fig. 8).

Permeability elliptic of 3D tensor permeability

3D tensor permeability of anisotropic formation in $x^{\prime}-y^{\prime}-z^{\prime}$ co-ordinate system is expressed as $\left[k^{\prime}\right]_{3 \times 3}$, permeability tensor in $x-y-z$ co-ordinate system is expressed as $[k]_{3 \times 3}$, and the angles between the two co-ordinate systems are fracture dip $\alpha$ and fracture azimuth $\beta$ (Liu et al. 2011). If the $x-y-z$ co-ordinate system is parallel to the direction of the permeability principal values, we can get the expression of $\left[k^{\prime}\right]_{3 \times 3}$.

$\left[k^{\prime}\right]_{3 \times 3}=[T]_{3 \times 3}[k]_{3 \times 3}\left[T^{\prime}\right]_{3 \times 3}$

where

$$
\begin{aligned}
{[T]_{3 \times 3} } & =\left[\begin{array}{ccc}
\cos \alpha \cos \beta & \sin \beta & -\sin \alpha \cos \beta \\
-\cos \alpha \sin \beta & \cos \beta & \sin \alpha \sin \beta \\
\sin \alpha & 0 & \cos \alpha
\end{array}\right] ;[k]_{3 \times 3} \\
& =\left[\begin{array}{ccc}
k_{x x} & 0 & 0 \\
0 & k_{y y} & 0 \\
0 & 0 & k_{z z}
\end{array}\right] .
\end{aligned}
$$




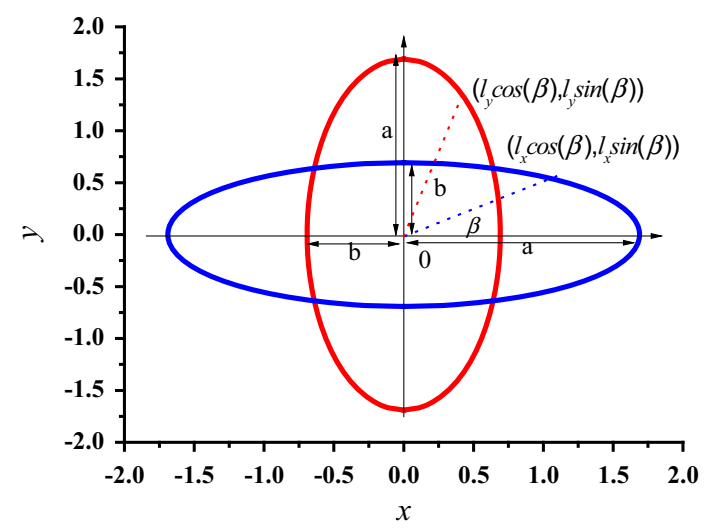

Fig. 7 Scheme of 2D seepage ellipse

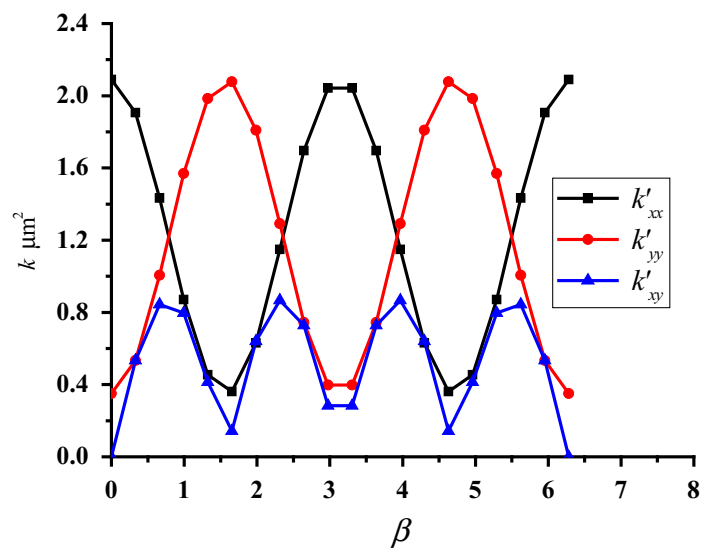

Fig. 8 The relationship between $\left[k^{\prime}\right]$ and the angle

The permeability tensor of each component can be expressed as

$$
\left\{\begin{array}{l}
k_{x x}^{\prime}=\cos ^{2} \alpha \cos ^{2} \beta k_{x x}+\sin ^{2} \beta k_{y y}+\sin ^{2} \alpha \cos ^{2} \beta k_{z z} \\
k_{x y}^{\prime}=-\cos ^{2} \alpha \sin 2 \beta \frac{k_{x x}}{2}+\sin 2 \beta \frac{k_{y y}}{2}-\sin ^{2} \alpha \sin 2 \beta \frac{k_{z z}}{2} \\
k_{x z}^{\prime}=\sin 2 \alpha \cos \beta \frac{k_{x x}-k_{z z}}{2} \\
k_{y y}^{\prime}=\cos ^{2} \alpha \sin ^{2} \beta k_{x x}+\cos ^{2} \beta k_{y y}+\sin ^{2} \alpha \sin ^{2} \beta k_{z z} \\
k_{y z}^{\prime}=\sin 2 \alpha \sin \beta \frac{k_{z z}-k_{x x}}{2} \\
k_{z z}^{\prime}=\sin ^{2} \alpha k_{x x}+\cos ^{2} \alpha k_{z z} .
\end{array}\right.
$$

So we can get permeability elliptic of 3D tensor permeability in principal direction

$$
\begin{aligned}
& \frac{\left(l_{x} \cos \alpha \cos \beta\right)^{2}}{a^{2}}+\frac{\left(l_{x} \sin \beta\right)^{2}}{b^{2}}+\frac{\left(l_{x} \sin \alpha \cos \beta\right)^{2}}{c^{2}}=1 \\
& \frac{\left(l_{y} \cos \alpha \sin \beta\right)^{2}}{a^{2}}+\frac{\left(l_{y} \cos \beta\right)^{2}}{b^{2}}+\frac{\left(l_{y} \sin \alpha \sin \beta\right)^{2}}{c^{2}}=1
\end{aligned}
$$

$\frac{\left(l_{z} \sin \alpha\right)^{2}}{a^{2}}+\frac{\left(l_{z} \cos \alpha\right)^{2}}{c^{2}}=1$

where $\quad a=\sqrt{\frac{1}{k_{x x}}} ; b=\sqrt{\frac{1}{k_{y y}}} ; c=\sqrt{\frac{1}{k_{z z}}} ; l_{x}=\sqrt{\frac{1}{k_{x x}^{\prime}}} ; l_{y}=$ $\sqrt{\frac{1}{k_{y y}^{\prime}}} ; l_{z}=\sqrt{\frac{1}{k_{z z}^{\prime}}}$.

Assuming $[k]_{3 \times 3}=\left[\begin{array}{ccc}5.07 & 0 & 0 \\ 0 & 0.84 & 0 \\ 0 & 0 & 1.65\end{array}\right]$, we can get the distribution of $\left[k^{\prime}\right]_{3 \times 3}$ in $x^{\prime}-y^{\prime}-z^{\prime}$ co-ordinate system. And the distribution of permeability is shown in Fig. 9.

\section{The characteristics of fractured media anisotropy}

If there is single group parallel fracture developed in reservoir unit, the permeability tensor of fracture and matrix is $\left[k_{f}\right]$ and $\left[k_{m}\right]$, respectively. The $x-y-z$ co-ordinate system is established along the fracture direction, reservoir 3D tensor permeability is

$$
\begin{aligned}
{[k] } & =\left[k_{\mathrm{f}}\right]+\left[k_{\mathrm{m}}\right]=\left[\begin{array}{lll}
k_{\mathrm{fr}} & & \\
& 0 & \\
& & 0
\end{array}\right]+\left[\begin{array}{lll}
k_{\mathrm{ma}} & & \\
& k_{\mathrm{ma}} & \\
& & k_{\mathrm{ma}}
\end{array}\right] \\
& =\left[\begin{array}{lll}
k_{\mathrm{fr}}+k_{\mathrm{ma}} & & \\
& k_{\mathrm{ma}} & \\
& & k_{\mathrm{ma}}
\end{array}\right] .
\end{aligned}
$$

Combining Eqs. (26) and (31), we can get the permeability tensor in $x^{\prime}-y^{\prime}-z^{\prime}$ reference co-ordinate system, and its expression is

$$
\left[k^{\prime}\right]=\left[\begin{array}{ccc}
\cos ^{2} \alpha \cos ^{2} \beta k_{\mathrm{fr}}+k_{\mathrm{ma}} & -\frac{k_{\mathrm{fr}} \cos ^{2} \alpha \sin 2 \beta}{2} & \sin 2 \alpha \cos \beta \frac{k_{\mathrm{fr}}}{2} \\
-\frac{k_{\mathrm{fr}} \cos ^{2} \alpha \sin 2 \beta}{2} & k_{\mathrm{fr}} \cos ^{2} \alpha \sin ^{2} \beta+k_{\mathrm{ma}} & -\sin 2 \alpha \sin \beta \frac{k_{\mathrm{fr}}}{2} \\
\sin 2 \alpha \cos \beta \frac{k_{\mathrm{fr}}}{2} & -\sin 2 \alpha \sin \beta \frac{k_{\mathrm{fr}}}{2} & \sin ^{2} \alpha k_{\mathrm{fr}}+k_{\mathrm{ma}}
\end{array}\right] .
$$

It is well known that $k_{\mathrm{ma}}$ is much less than $k_{\mathrm{fr}}$, so the Eq. (32) can be simplified to formula (33)

$\left[k^{\prime}\right]=k_{\mathrm{fr}}\left[\begin{array}{ccc}\cos ^{2} \alpha \cos ^{2} \beta & -\frac{\cos ^{2} \alpha \sin 2 \beta}{2} & \frac{\sin 2 \alpha \cos \beta}{2} \\ -\frac{\cos ^{2} \alpha \sin 2 \beta}{2} & \cos ^{2} \alpha \sin ^{2} \beta & \frac{-\sin 2 \alpha \sin \beta}{2} \\ \frac{\sin 2 \alpha \cos \beta}{2} & \frac{-\sin 2 \alpha \sin \beta}{2} & \sin ^{2} \alpha\end{array}\right]$.

If there are $N$ fracture groups developed in the reservoir unit, the permeability tensors of the $i$ th $(1 \leq i \leq N)$ group of fracture in the $x-y-z$ co-ordinate system can be written as

$\left[k_{i}\right]=\left[\begin{array}{lll}k_{x x i} & k_{x y i} & k_{x z i} \\ k_{y x i} & k_{y y i} & k_{y z i} \\ k_{z x i} & k_{z y i} & k_{z z i}\end{array}\right]$

According to the superposition principle, the whole permeability tensor can be derived 

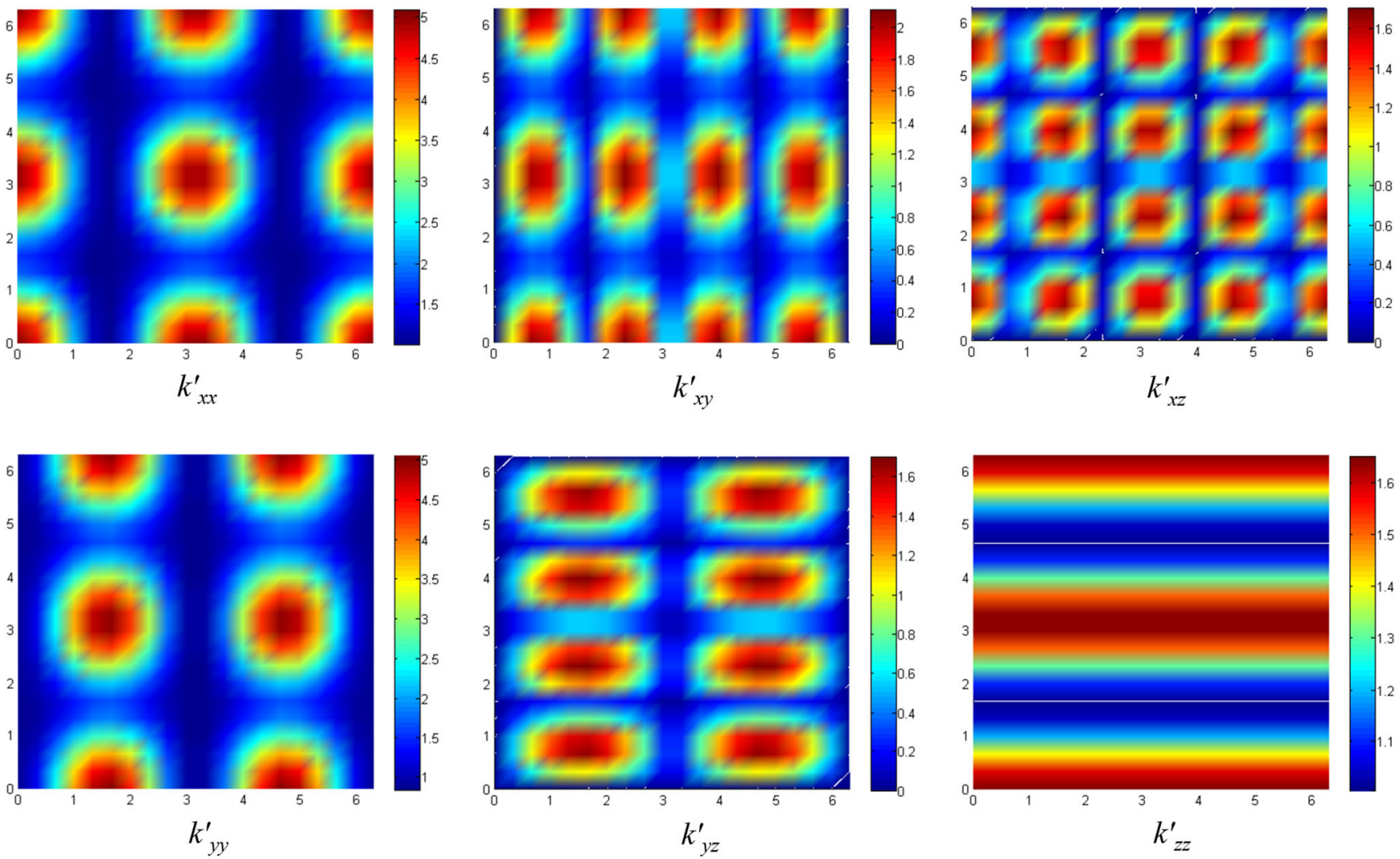

Fig. 9 The distribution of permeability tensor in $x^{\prime}-y^{\prime}-z^{\prime}$ co-ordinate system

$$
[k]=\sum_{i=1}^{N}\left[k_{i}\right]=\left[\begin{array}{lll}
\sum_{i=1}^{N} k_{x x i} & \sum_{i=1}^{N} k_{x y i} & \sum_{i=1}^{N} k_{x z i} \\
\sum_{i=1}^{N} k_{y x i} & \sum_{i=1}^{N} k_{y y i} & \sum_{i=1}^{N} k_{y z i} \\
\sum_{i=1}^{N} k_{z x i} & \sum_{i=1}^{N} k_{z y i} & \sum_{i=1}^{N} k_{z z i}
\end{array}\right] .
$$

If there are $N$ groups of paralleling fracture developed in reservoir unit, where fracture permeability is $k_{\text {fri }}$, matrix permeability is $k_{\mathrm{ma}}$ and the fracture dip and fracture azimuth of the $i$ th group fracture is $\alpha_{i}$ and $\beta_{i}$, the equivalent permeability tensor of the reservoir is shown below according to Eqs. (33) and (35).

$[k]=\sum_{i=1}^{N}\left[\begin{array}{ccc}k_{\mathrm{fri}} \cos ^{2} \alpha_{i} \cos ^{2} \beta_{i}+k_{\mathrm{ma}} & -\frac{k_{\mathrm{fri}} \cos ^{2} \alpha_{i} \sin 2 \beta_{i}}{2} & \frac{k_{\mathrm{fin}} \sin 2 \alpha_{i} \cos \beta_{i}}{2} \\ -\frac{k_{\mathrm{fri}} \cos ^{2} \alpha_{i} \sin 2 \beta_{i}}{2} & k_{\mathrm{fri}} \cos ^{2} \alpha_{i} \sin ^{2} \beta_{i}+k_{\mathrm{ma}} & \frac{-k_{\mathrm{fin}} \sin 2 \alpha_{i} \sin \beta_{i}}{2} \\ \frac{k_{\mathrm{fi}} \sin 2 \alpha_{i}^{2} \cos \beta_{i}}{2} & \frac{-k_{\mathrm{fri}} \sin 2 \alpha_{i} \sin \beta_{i}}{2} & k_{\mathrm{fri}} \sin ^{2} \alpha_{i}+k_{\mathrm{ma}}\end{array}\right]$

Since $k_{\mathrm{ma}}$ is far less than $k_{\text {fri }}$, Eq. (36) can be simplified to

$[k]=\sum_{i=1}^{N}\left[\begin{array}{ccc}k_{\mathrm{fri}} \cos ^{2} \alpha_{i} \cos ^{2} \beta_{i} & -\frac{k_{\mathrm{fri}} \cos ^{2} \alpha_{i} \sin 2 \beta_{i}}{2} & \frac{k_{\mathrm{fri}} \sin 2 \alpha_{i} \cos \beta_{i}}{2} \\ -\frac{k_{\mathrm{fri}} \cos ^{2} \alpha_{i} \sin 2 \beta_{i}}{2} & k_{\mathrm{fri}} \cos ^{2} \alpha_{i} \sin \beta^{2} \beta_{i} & -\frac{k_{\mathrm{fri}} \sin 2 \alpha_{i} \sin \beta_{i}}{2} \\ \frac{k_{\mathrm{fri}} \sin 2 \alpha_{i} \cos \beta_{i}}{2} & \frac{-k_{\mathrm{fri}} \sin 2 \alpha_{i} \sin \beta_{i}}{2} & k_{\mathrm{fri}} \sin ^{2} \alpha_{i}\end{array}\right]$.
Permeability of fractures is strongly stress-dependent. The relationship between permeability and confining pressure is determined in (Gangi 1978), thus the formula considering the stress dependence can be written as

$k_{\mathrm{fr}}=k_{\mathrm{fr} 0}\left[1-\left(p / p_{1}\right)^{m}\right]^{3}$

where $k_{f r 0}$ is fracture permeability under the initial pressure $p_{0}, \mu \mathrm{m}^{2} ; m$ is a constant $(0<m<1)$ and it characterizes the distribution of the asperity length; $p_{1}$ is the effective modulus of the asperities, MPa; $p$ is the current pressure, MPa. According to Eqs. (33) and (38), the full tensor permeability expression of single fracture group in fractured media can be obtained as

$[k]=k_{\mathrm{fr} 0}\left[1-\left(p / p_{1}\right)^{m}\right]^{3}\left[\begin{array}{ccc}\cos ^{2} \alpha \cos ^{2} \beta & -\frac{\cos ^{2} \alpha \sin 2 \beta}{2} & \frac{\sin 2 \alpha \cos \beta}{2} \\ -\frac{\cos ^{2} \alpha \sin 2 \beta}{2} & \cos ^{2} \alpha \sin ^{2} \beta & \frac{-\sin 2 \alpha \sin \beta}{2} \\ \frac{\sin 2 \alpha \cos \beta}{2} & \frac{-\sin 2 \alpha \sin \beta}{2} & \sin ^{2} \alpha\end{array}\right]$.

And the full tensor permeability of $N$ fracture groups can be obtained

$[k]=\left[\begin{array}{lll}k_{11} & k_{12} & k_{13} \\ k_{21} & k_{22} & k_{23} \\ k_{31} & k_{32} & k_{33}\end{array}\right]$ 


$$
\begin{aligned}
& k_{11}=\sum_{i=1}^{N} k_{\mathrm{fr} 0 i}\left[1-\left(p / p_{1 i}\right)^{m_{i}}\right]^{3} \cos ^{2} \alpha_{i} \cos ^{2} \beta_{i} ; \\
& k_{12}=k_{21}=-\sum_{i=1}^{N} \frac{k_{\mathrm{fr} 0 i}\left[1-\left(p / p_{1 i}\right)^{m_{i}}\right]^{3} \cos ^{2} \alpha_{i} \sin 2 \beta_{i}}{2} \\
& k_{13}=k_{31}=\sum_{i=1}^{N} \frac{k_{\mathrm{fr} 0 i}\left[1-\left(p / p_{1 i}\right)^{m_{i}}\right]^{3} \sin 2 \alpha_{i} \cos \beta_{i}}{2} \text {; } \\
& k_{22}=\sum_{i=1}^{N} k_{\mathrm{fr} 0 i}\left[1-\left(p / p_{1 i}\right)^{m_{i}}\right]^{3} \cos ^{2} \alpha_{i} \sin ^{2} \beta_{i} \\
& k_{23}=k_{32}=-\sum_{i=1}^{N} \frac{k_{\mathrm{fr} 0 i}\left[1-\left(p / p_{1 i}\right)^{m_{i}}\right]^{3} \sin 2 \alpha_{i} \sin \beta_{i}}{2} ; \\
& k_{33}=\sum_{i=1}^{N} k_{\mathrm{fr} 0 i}\left[1-\left(p / p_{1 i}\right)^{m_{i}}\right]^{3} \sin ^{2} \alpha_{i}
\end{aligned}
$$

where $k_{\mathrm{fr} O i}$ is initial permeability of the $i$ th fracture group, $\mu \mathrm{m}^{2} ; m_{i}$ is the deformation coefficient of the $i$ th fracture group; $\alpha_{i}$ is fracture dip of the $i$ th fracture group; $\beta_{i}$ is fracture azimuth of the $i$ th fracture group; $N$ is the number of fracture groups.

\section{Conclusions}

1. Based on percolation characteristics of anisotropy fractured media, a new 2D tensor permeability test method in laboratory is established. The developed model introduces an exceptional and accurate experimental procedure integrated with a simple mathematical calculation to measure 2D tensor permeability for anisotropic fractured media.

2. Combining numerical simulation results and flow experiment results in $x$ and $y$ direction, the 2D tensor permeability is derived. And the variation mechanism of full tensor permeability in fractured anisotropic media is revealed.

3. According to the polar form of elliptic equation, permeability elliptic is derived. With permeability elliptic, the change law of permeability value in principal direction is studied.

4. Based on the quantitative characterization of $3 \mathrm{D}$ tensor permeability and Gangi's permeability stress sensitivity model, a new 3D Permeability tensor mathematical model for fractured media is derived.

Open Access This article is distributed under the terms of the Creative Commons Attribution License which permits any use, distribution, and reproduction in any medium, provided the original author(s) and the source are credited.

\section{References}

Asadi M, Ghalambor A, Rose WD et al (2000) Anisotropic permeability measurement of porous media: a 3-Dimensional method. Soc Petrol Eng 59396

Bagheri M, Settari A (2007) Methods for modelling full tensor permeability in reservoir simulators. J Can Petrol Technol 46(3):31-38

Chapuis RP, Gill DE (1989) Hydraulic anisotropy of homogeneous soils and rocks: influence of the densification process. Bull Eng Geol Environ 39(1):75-86

Chen HY, Hidayati DT, Lawrence W (1998) Teufel, estimation of permeability anisotropy and stress anisotropy from interference testing. Soc Petrol Eng 49235

Dayani S, Baghbanan A, Karami M (2012) Evaluating the permeability tensor of a fractured rock mass using effective medium theory. In: Eurock 2012, the 2012 ISRM International symposium rock engineering and technology for sustainable underground construction

Ferrandon J (1948) Les lois d'écoulement de filtration. Le Génie civil 125(2):24-28

Gangi AF (1978) Variation of whole and fractured porous rock permeability with confining pressure. Int J Rock Mech Min Sci Geomech Abstr 15(5):249-257

Greenkorn RA, Johnson CR, Shallenberger LK (1964) Directional permeability of heterogeneous anisotropic porous media. Soc Petrol Eng J 4(2):124-132

Hassanpour RM, Leuangthong O, Deutsch CV (2008) Calculation of permeability tensors for unstructured grid blocks. Petrol Soc J

Johnson WE, Breston NJ (1951) Directional permeability measurements on oil sandstone from various states. Prod Mon 14(4):10-19

Johnson WE, Hughes RV (1948) Directional permeability measurements and their significance. Prod Mon 13(1):17-25

Leung WF (1986) A tensor model for anisotropic and heterogeneous reservoirs with variable directional permeabilities [R]. Soc Petrol Eng 15134:405-420

Liakopoulos AC (1960) Variation of the permeability tensor ellipsoid in homogenous anisotropic soils. Civil Engineering Department, College of Engineering, Riyadh

Liakopoulos AC (1965a) Darcy's coefficient of permeability as symmetric tensor of second rank. Bull Int Assoc Sci Hydrol 10(3):41-48

Liakopoulos AC (1965b) Variation of the permeability tensor ellipsoid in homogeneous anisotropic soils. Water Resour Res 1(1):135-141

Liu YT, Ding ZP, Qu YG et al (2011) The characterization of fracture orientation and the calculation of anisotropic permeability parameters of reservoirs. Acta Petrolei Sinica 32(5):842-846

Ma CY, Liu YT, Wu JL (2013) Simulated flow model of fractured anisotropic media: permeability and fracture. Theor Appl Fract Mech 65:28-33

Marcus H (1962) The permeability of a sample of an anisotropic porous media. J Geophys Res 67(13):5215-5225

Masland M, Kirkham D (1955) Theory and measurement of anisotropic air permeability in soil. Soil Sci Soc Am 19(4):395-400

Metwally Y, Chesnokov EM (2010) Measuring gas shale permeability tensor in the lab scale. SEG Denver 2010 Annual Meeting, pp 2628-2633

Mina KB, Rutqvist J, Tsang CF et al (2004) Stress-dependent permeability of fractured rock masses: a numerical study. Int $\mathbf{J}$ Rock Mech Min Sci 41:1191-1210

Oda M (1985) Permeability tensor for discontinuous rock masses. Geotechnique 35(4):483-495 
Parsons RW (1964) Directional permeability of heterogeneous anisotropic porous media: discussion. Soc Petrol Eng J 4(4): 363-364

Renard P, Genty A, Stauffer F (2001) Laboratory determination of the full permeability tensor. J Geophys Res 106:442-452

Rong G, Peng J, Wang X et al (2013) Permeability tensor and representative elementary volume of fractured rock masses. Hydrogeol J 21:1655-1671

Scheidegger AE (1954) Directional permeability of porous media to homogeneous fluids. Geofisica pura e applicata 28(1):75-90
Snow DT (1969) Anisotropic permeability of fractured media. Water Resour Res 5(6):1273-1289

Wong RCK, Du J (2003) Application of strain-induced permeability model in a coupled geomechanics-reservoir simulator. Paper No. 2003-149. Petroleum Society, Canadian Institute of Mining, Metallurgy and Petroleum

Wong RCK, Li Y (2001) A deformation-dependent model for permeability changes in oil sand due to shear dilation. J Can Pet Technol 4(8):37-44 